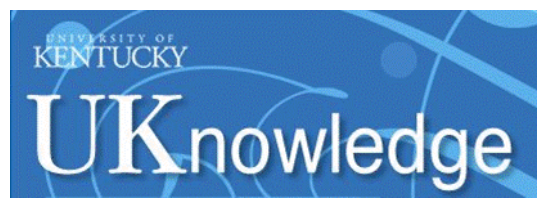

University of Kentucky

UKnowledge

$11-30-2016$

\title{
SDSS-IV MaNGA: A Serendipitous Observation of a Potential Gas Accretion Event
}

\author{
Edmond Cheung \\ The University of Tokyo, Japan \\ David V. Stark \\ The University of Tokyo, Japan \\ Song Huang \\ The University of Tokyo, Japan \\ Kate H. R. Rubin \\ Harvard-Smithsonian Center for Astrophysics \\ Lihwai Lin \\ Academia Sinica, Taiwan
}

See next page for additional authors

Follow this and additional works at: https://uknowledge.uky.edu/physastron_facpub

Part of the Astrophysics and Astronomy Commons, and the Physics Commons

Right click to open a feedback form in a new tab to let us know how this document benefits you.

\section{Repository Citation}

Cheung, Edmond; Stark, David V.; Huang, Song; Rubin, Kate H. R.; Lin, Lihwai; Tremonti, Christy; Zhang, Kai; Yan, Renbin; Bizyaev, Dmitry; and Boquien, Médéric, "SDSS-IV MaNGA: A Serendipitous Observation of a Potential Gas Accretion Event" (2016). Physics and Astronomy Faculty Publications. 496.

https://uknowledge.uky.edu/physastron_facpub/496

This Article is brought to you for free and open access by the Physics and Astronomy at UKnowledge. It has been accepted for inclusion in Physics and Astronomy Faculty Publications by an authorized administrator of UKnowledge. For more information, please contact UKnowledge@lsv.uky.edu. 
SDSS-IV MaNGA: A Serendipitous Observation of a Potential Gas Accretion Event

Digital Object Identifier (DOI)

https://doi.org/10.3847/0004-637X/832/2/182

Notes/Citation Information

Published in The Astrophysical Journal, v. 832, no. 2, 182, p. 1-10.

(C) 2016. The American Astronomical Society. All rights reserved.

The copyright holder has granted the permission for posting the article here.

Authors

Edmond Cheung, David V. Stark, Song Huang, Kate H. R. Rubin, Lihwai Lin, Christy Tremonti, Kai Zhang, Renbin Yan, Dmitry Bizyaev, and Médéric Boquien 


\title{
SDSS-IV MaNGA: A SERENDIPITOUS OBSERVATION OF A POTENTIAL GAS ACCRETION EVENT
}

\author{
Edmond Cheung ${ }^{1}$, David V. Stark ${ }^{1}$, Song Huang ${ }^{1}$, Kate H. R. Rubin ${ }^{2}$, Lihwai Lin ${ }^{3}$, Christy Tremonti ${ }^{4}$, Kai $_{\text {Zhang }}{ }^{5}$, \\ Renbin Yan ${ }^{5}$, Dmitry Bizyaev ${ }^{6,7}$, Médéric Boquien ${ }^{8}$, Joel R. Brownstein ${ }^{9}$, Niv Drory ${ }^{10}$, Joseph D. Gelfand ${ }^{11,12}$, \\ Johan H. Knapen ${ }^{13,14}$, Roberto Maiolino ${ }^{15,16}$, Olena Malanushenko ${ }^{6}$, Karen L. Masters ${ }^{17}$, Michael R. Merrifield ${ }^{18}$, \\ Zach Pace ${ }^{4}$, Kaike Pan ${ }^{6}$, Rogemar A. Riffel ${ }^{19,20}$, Alexandre Roman-Lopes ${ }^{21}$, Wiphu Rujopakarn ${ }^{1,22}$, \\ Donald P. Schneider ${ }^{23,24}$, John P. StotT ${ }^{25}$, Daniel Thomas ${ }^{17}$, and AnNe-Marie Weijmans ${ }^{26}$ \\ ${ }^{1}$ Kavli Institute for the Physics and Mathematics of the Universe (WPI), The University of Tokyo Institutes for Advanced Study, The University of Tokyo, Kashiwa, \\ Chiba 277-8583, Japan; ec2250@gmail.com \\ ${ }^{2}$ Harvard-Smithsonian Center for Astrophysics, 60 Garden Street, Cambridge, MA 02138, USA \\ ${ }^{3}$ Institute of Astronomy and Astrophysics, Academia Sinica, Taipei 106, Taiwan \\ ${ }^{4}$ Department of Astronomy, University of Wisconsin-Madison, 475 North Charter Street, Madison, WI 53706, USA \\ ${ }^{5}$ Department of Physics and Astronomy, University of Kentucky, 505 Rose Street, Lexington, KY 40506-0055, USA \\ ${ }^{6}$ Apache Point Observatory and New Mexico State University, P.O. Box 59, Sunspot, NM, 88349-0059, USA \\ ${ }^{7}$ Sternberg Astronomical Institute, Moscow State University, Moscow, Russia \\ ${ }^{8}$ Unidad de Astronomía, Universidad de Antofagasta, Avenida Angamos 601, Antofagasta 1270300, Chile \\ ${ }^{9}$ Department of Physics and Astronomy, University of Utah, 115 S. 1400 E., Salt Lake City, UT 84112, USA \\ ${ }^{10}$ McDonald Observatory, Department of Astronomy, University of Texas at Austin, 1 University Station, Austin, TX 78712-0259, USA \\ ${ }^{11}$ NYU Abu Dhabi, P.O. Box 129188, Abu Dhabi, UAE \\ ${ }^{12}$ Center for Cosmology and Particle Physics, New York University, Meyer Hall of Physics, 4 Washington Place, New York, NY 10003, USA \\ ${ }^{13}$ Instituto de Astrofísica de Canarias, E-38205 La Laguna, Tenerife, Spain \\ ${ }^{14}$ Departamento de Astrofísica, Universidad de La Laguna, E-38205 La Laguna, Tenerife, Spain \\ ${ }_{16}$ Cavendish Laboratory, University of Cambridge, 19 J. J. Thomson Avenue, Cambridge CB3 OHE, UK \\ ${ }^{16}$ Kavli Institute for Cosmology, University of Cambridge, Madingley Road, Cambridge CB3 OHA, UK \\ ${ }^{17}$ Institute for Cosmology and Gravitation, University of Portsmouth, Dennis Sciama Building, Burnaby Road, Portsmouth PO1 3FX, UK \\ ${ }^{18}$ School of Physics and Astronomy, University of Nottingham, University Park, Nottingham, NG7 2RD, UK \\ ${ }^{19}$ Departamento de Física, Centro de Cien̂cias Naturais e Exatas, Universidade Federal de Santa Maria, 97105-900 Santa Maria, RS, Brazil \\ ${ }^{20}$ Laboratório Interinstitucional de e-Astronomia-LIneA, Rua Gal. José Cristino 77, Rio de Janeiro, RJ-20921-400, Brazil \\ ${ }^{21}$ Departamento de Física y Astronomía, Facultad de Ciencias, Universidad de La Serena, Cisternas 1200, La Serena, Chile \\ 22 Department of Physics, Faculty of Science, Chulalongkorn University, 254 Phayathai Road, Pathumwan, Bangkok 10330, Thailand \\ ${ }_{23}$ Department of Astronomy and Astrophysics, The Pennsylvania State University, University Park, PA 16802, USA \\ ${ }^{24}$ Institute for Gravitation and the Cosmos, The Pennsylvania State University, University Park, PA 16802, USA \\ ${ }^{25}$ Sub-department of Astrophysics, Department of Physics, University of Oxford, Denys Wilkinson Building, Keble Road, Oxford OX1 3RH, UK \\ ${ }^{26}$ School of Physics and Astronomy, University of St Andrews, North Haugh, St Andrews, Fife KY16 9SS, UK \\ Received 2016 June 17; revised 2016 September 20; accepted 2016 September 30; published 2016 November 30
}

\section{ABSTRACT}

The nature of warm, ionized gas outside of galaxies may illuminate several key galaxy evolutionary processes. A serendipitous observation by the MaNGA survey has revealed a large, asymmetric $\mathrm{H} \alpha$ complex with no optical counterpart that extends $\approx 8^{\prime \prime}(\approx 6.3 \mathrm{kpc})$ beyond the effective radius of a dusty, starbursting galaxy. This $\mathrm{H} \alpha$ extension is approximately three times the effective radius of the host galaxy and displays a tail-like morphology. We analyze its gas-phase metallicities, gaseous kinematics, and emission-line ratios and discuss whether this $\mathrm{H} \alpha$ extension could be diffuse ionized gas, a gas accretion event, or something else. We find that this warm, ionized gas structure is most consistent with gas accretion through recycled wind material, which could be an important process that regulates the low-mass end of the galaxy stellar mass function.

Key words: galaxies: abundances - galaxies: evolution - galaxies: formation - galaxies: starburst

\section{INTRODUCTION}

Understanding the warm, ionized gas outside of galaxies is a critical aspect of galaxy evolution. To study this gas, there have been two main probes: (1) observations of extraplanar ionized gas in edge-on galaxies (e.g., Rand et al. 1990; Tüllmann et al. 2000; Otte et al. 2001; Miller \& Veilleux 2003; Rossa \& Dettmar 2003) and (2) low-ionization metal-line absorption studies using quasar sightlines (e.g., Tumlinson et al. 2011; Werk et al. 2013, 2014).

These studies have led to the discovery of diffuse ionized gas (DIG; Hoyle \& Ellis 1963; Reynolds 1985), which is a layer of warm, low-density ionized gas that extends out to several kiloparsecs into the halos of galaxies, and the confirmation of the circumgalactic medium (CGM; Bergeron 1986; Lanzetta et al. 1995), which is a gas reservoir containing warm, ionized gas that is of even lower density than the DIG and extends hundreds of kiloparsecs into the halos of galaxies. But how this gas relates to the evolution of their host galaxies is an open question.

In this work, we further our understanding of warm, ionized gas in the halos of galaxies by studying a rare and unusual gas complex in the SDSS-IV MaNGA survey (Bundy et al. 2015). Designed to observe galaxies out to a maximum radius of 2.5 effective radii $\left(R_{\mathrm{e}}\right)$, the MaNGA survey has observed a dusty, starbursting ${ }^{27}$ galaxy that is on the upper end of the mass-metallicity relationship (Tremonti et al. 2004) out to $6.3 R_{\mathrm{e}}$ through a fortuitous overestimation

\footnotetext{
$\overline{{ }^{27} \text { We define starbursts as galaxies with }} \log \Sigma_{\mathrm{SFR}}>-1 M_{\odot} \mathrm{yr}^{-1} \mathrm{kpc}^{-2}$ (Kennicutt \& Evans 2012).
} 
of $R_{\mathrm{e}}{ }^{28}$ This galaxy shows no signs of interaction and displays a large $\mathrm{H} \alpha$ extension with no optical counterpart in the Sloan Digital Sky Survey Data Release 7 (SDSS DR7; York et al. 2000; Abazajian et al. 2009).

Throughout this work, we assume a flat cosmological model with $H_{0}=70 \mathrm{~km} \mathrm{~s}^{-1} \mathrm{Mpc}^{-1}, \Omega_{m}=0.30$, and $\Omega_{\Lambda}=0.70$.

\section{DATA}

The data used in this work are from the ongoing SDSS-IV MaNGA survey (Bundy et al. 2015; Drory et al. 2015; Law et al. 2015; SDSS Collaboration et al. 2016; Yan et al. 2016), which is an integral field unit (IFU) survey that is taking resolved spectroscopy of 10,000 nearby galaxies with $\log M_{*} /$ $M_{\odot} \gtrsim 9$. The survey uses the SDSS $2.5 \mathrm{~m}$ telescope (Gunn et al. 2006) and BOSS spectrographs (Smee et al. 2013); pilot studies using P-MaNGA data include $\mathrm{Li}$ et al. (2015), Wilkinson et al. (2015), and Belfiore et al. (2015). The $r$-band signal-to-noise ratio $(\mathrm{S} / \mathrm{N})$ in the galaxy outskirts is $4-8 \AA^{-1}$, and the wavelength coverage is $3600-10000 \AA$. The effective spatial resolution is 2".5(FWHM), with an instrumental resolution of $\sigma \approx 60 \mathrm{~km} \mathrm{~s}^{-1}$ and a spectral resolution of $R \sim 2000$.

The MaNGA sample and data products were drawn from the internal MaNGA Product Launch-4 (MPL-4), which contains 1368 galaxies. Ancillary data are from the MPA-JHU DR7 value-added catalog ${ }^{29}$ and the NASA-Sloan Atlas. ${ }^{30}$

The stellar masses from the MPA-JHU DR7 value-added catalog are estimated by fitting a large grid of stellar population models from Bruzual \& Charlot (2003) to the ugriz SDSS photometry, following the philosophy of Kauffmann et al. (2003) and Salim et al. (2007). The fiber star formation rates from the MPA-JHU DR7 value-added catalog are estimated using the technique described in Brinchmann et al. (2004), where galaxies with emission lines are fitted with the models of Charlot \& Longhetti (2001). The fiber star formation rate surface density is calculated over the 3 " diameter SDSS fiber.

The stellar continuum of each spaxel is fit by the MaNGA Data Analysis Pipeline (DAP; K. Westfall et al. 2016, in preparation), which uses PPXF (Cappellari \& Emsellem 2004) and the MIUSCAT stellar population models (Vazdekis et al. 2012). Although the MIUSCAT templates are built to reflect simple stellar populations, the mix of templates used to construct the best-fit stellar continuum does not represent a physically motivated stellar population.

Emission-line fluxes are measured through simple fluxsumming after subtraction of the stellar continuum (where there is stellar continuum). The wavelength passbands over which they are integrated are similar to that of Yan et al. (2006), ranging from 10 to $20 \AA$ around the central wavelength. For spectra that do not have measured stellar continua, we subtract a baseline continuum that is based on a linear fit to the red and blue sidebands; these sidebands extend approximately $100 \AA$ beyond the central passbands and have been chosen to avoid other strong emission lines.

\footnotetext{
${ }^{28}$ MaNGA uses $R_{\mathrm{e}}$ measurements from the NASA-Sloan Atlas, which estimated $R_{\mathrm{e}}=7$ !' 4 for this galaxy. After masking out the bright, nearby stars, we used GALFIT (Peng et al. 2002) to fit a single Sérsic model to this galaxy, yielding $R_{\mathrm{e}}=2$ !'6; see Section 2

29 http://www.mpa-garching.mpg.de/SDSS/DR7/

30 http://www.nsatlas.org
}

We adopt the nonparametric quantities $v_{\text {peak }}$ and $W_{80}$ to characterize the center (with respect to the systemic velocity of the host galaxy) and width (that contains $80 \%$ of the flux) of the $\mathrm{H} \alpha$ emission line (e.g., Harrison et al. 2014). We only measure $v_{\text {peak }}$ and $W_{80}$ in spaxels where the $\mathrm{H} \alpha$ flux has $\mathrm{S} / \mathrm{N}>5$.

To estimate the gaseous metallicities, $12+\log (\mathrm{O} / \mathrm{H})$, we use the IZI code (Blanc et al. 2015), which estimates the ionization parameter and gaseous metallicity based on a Bayesian analysis of a model grid with a set of input line fluxes; we used the Dopita et al. (2013) model grids, but we obtain similar results to those of Levesque et al. (2010). We provide [O II] $\lambda \lambda 3727,3729, \quad \mathrm{H} \beta, \quad[\mathrm{O}$ III] $\lambda 5007, \quad \mathrm{H} \alpha$, [N II] $\lambda 6583$, and [S II] $\lambda \lambda 6717,6731$; all provided line fluxes have $\mathrm{S} / \mathrm{N}>3$. Throughout this work, we correct for reddening using the Balmer decrement and the Fitzpatrick (1999) extinction law. The typical uncertainties of these IZI metallicities are $0.1-0.3$ dex.

We also estimate the gaseous metallicity using the [N II]/ [O II] calibration from Kewley \& Dopita (2002), which is relatively insensitive to variations in ionization parameter and is least sensitive to DIG contamination (Zhang et al. 2016, submitted). We estimate the uncertainties by adding in quadrature the intrinsic scatter of this calibration ( 0.04 dex; see Kewley \& Dopita 2002) and the measurement error of $\log [\mathrm{N} \mathrm{II}] /[\mathrm{O}$ II] propagated through the calibration, resulting in typical uncertainties of $0.05-0.15$ dex.

\section{RESULTS}

Our main result is presented in Figure 1. The SDSS gri color image of the system is shown in Figure 1(a), with the MaNGA footprint outlined by the magenta hexagon. Figure 1(b) displays the $\mathrm{H} \alpha$ flux map of this system, with the $\mathrm{H} \alpha$ flux contours in light gray. There is a large extension in the $\mathrm{H} \alpha$ flux distribution that extends $\approx 8^{\prime \prime}(\approx 6.3 \mathrm{kpc})$ to the left (east) beyond the effective radius of the host galaxy and does not correspond to any optical source in SDSS. This striking feature is elucidated in Figure 1(c), where we present the $\mathrm{H} \alpha$ flux contours superimposed on the SDSS $r$-band image, with the blue circle marking the $R_{\mathrm{e}}$ of the host galaxy.

Figures 1(d) and (e) show the spectra centered on the $\mathrm{H} \alpha$ emission line of the highlighted spaxels in the $\mathrm{H} \alpha$ complex (which is 6!"67 away from the center) and in the center of the host galaxy, respectively. Both spectra possess strong emission from the [N II] doublet, $\mathrm{H} \alpha$, and the [S II] doublet, confirming the authenticity of this $\mathrm{H} \alpha$ extension and indicating the presence of ionized gas throughout the entire system. Stacking all the spectra within the green circle centered in the $\mathrm{H} \alpha$ extension of Figure 1(b) - which we will refer to as the " $\mathrm{H} \alpha$ circle"- does not reveal a significant continuum. Properties of the host galaxy are listed in Table 1.

Figure 2 presents the gaseous metallicities, $12+\log (\mathrm{O} / \mathrm{H})$, of this system using IZI (Figure 2(a)) and the [N II]/[O II] calibration (Figure 2(b)), with the $\mathrm{H} \alpha$ flux contours overlaid and the same spaxels from Figure 1(b) highlighted in white. Using the estimates of IZI (the $[\mathrm{N}$ II $] /[\mathrm{O}$ II $]$ calibration), the stacked $12+\log (\mathrm{O} / \mathrm{H})$ value in a 1.15 circle centered on the host galaxy is $9.06 \pm 0.06(8.92 \pm 0.02)$, whereas the stacked $12+\log (\mathrm{O} / \mathrm{H})$ value in the $\mathrm{H} \alpha$ circle is $8.81 \pm 0.05$ $(8.77 \pm 0.03)-0.25(0.15)$ dex less than the center of the host galaxy at greater than $99.7 \%$ confidence. Comparing these gaseous metallicities to the solar value $(12+\log (\mathrm{O} /$ $\mathrm{H})_{\odot}=8.69$; Asplund et al. 2009) reveals that the entire 


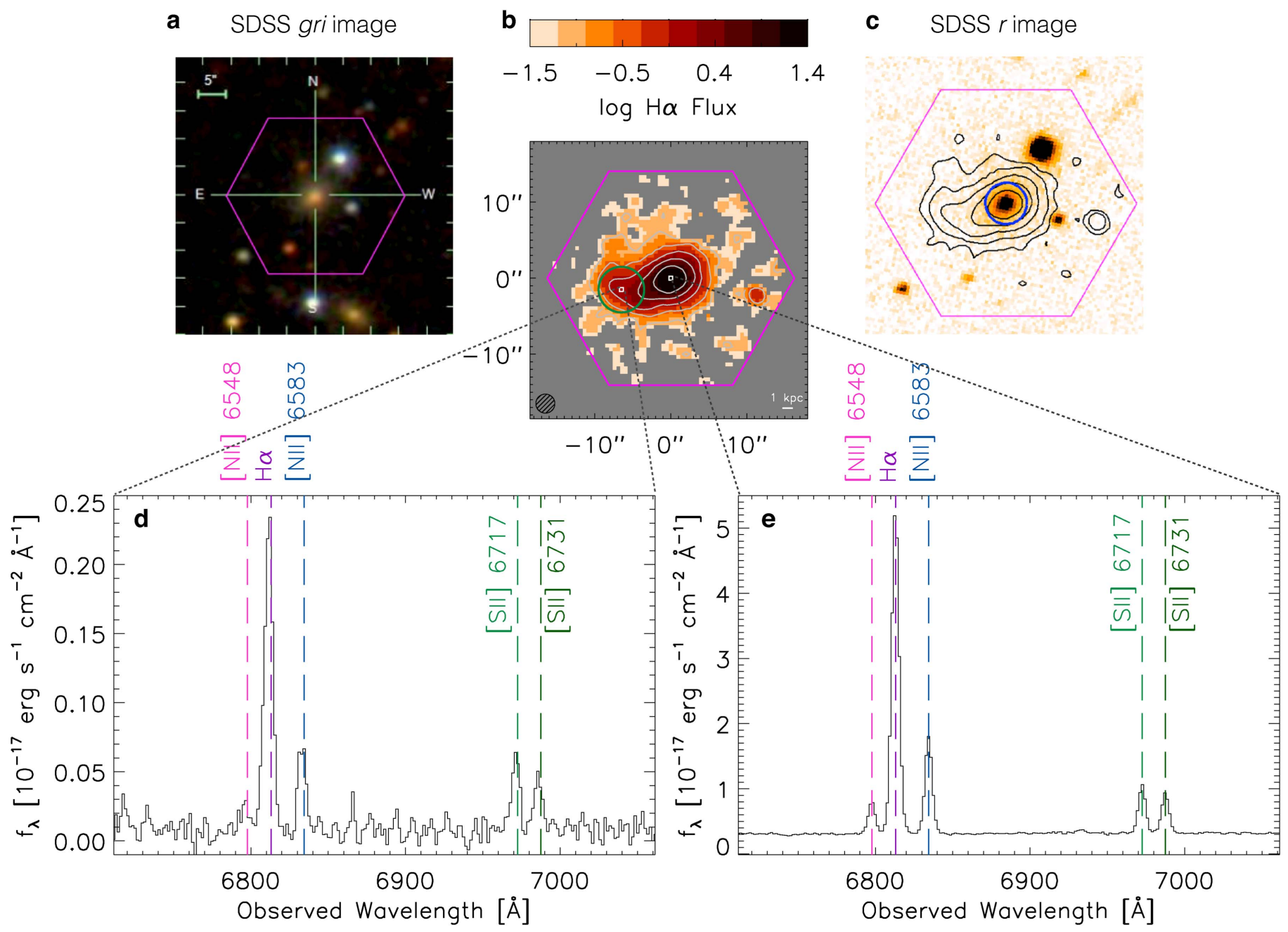

Figure 1. (a) SDSS gri color image of the object, with the MaNGA field of view in magenta. (b) $\mathrm{H} \alpha$ flux map with contours of log $\mathrm{H} \alpha \mathrm{Flux}=-1,-0.5,0,0.5$, and 1 ; the flux units are $10^{-17} \mathrm{erg} \mathrm{s}^{-1} \mathrm{~cm}^{-2}$. There is an asymmetric extension in the $\mathrm{H} \alpha$ flux distribution to the left (east) of the host galaxy. The green circle, which has a radius of $3^{\prime \prime}$, is an approximation of this $\mathrm{H} \alpha$ extension - we refer to this as the "H $\alpha$ circle" throughout the text. The lower left hatched circle represents the effective spatial resolution of MaNGA, FWHM $=2$ ". 5. (c) SDSS $r$-band image with the H $\alpha$ flux contours superimposed; the blue circle marks the $R_{\mathrm{e}}$ of the host galaxy. There is no optical source in the region of the $\mathrm{H} \alpha$ extension. (d, e) Spectra (before stellar continuum subtraction) from the indicated spaxels in the H $\alpha$ extension and in the center of the galaxy, respectively. The vertical, dashed lines indicate the expected wavelengths of the [N II] doublet, $\mathrm{H} \alpha$, and [S II] doublet emission lines at the systemic velocity of the host galaxy.

system has supersolar gaseous metallicities (but see Kennicutt et al. 2003 for caveats associated with strong-line abundances).

Figures 2(c) and (d) display the $12+\log (\mathrm{O} / \mathrm{H})$ profile as traced by the highlighted spaxels in Figures 2(a) and (b), with the characteristic metallicity profile of noninteracting disks from Sánchez et al. (2014) overplotted in the dashed line (with an arbitrary zero point). Out to $\sim 2 R_{\mathrm{e}}$, the metallicity profiles of this system are similar to that of Sánchez et al. (2014). At $\gtrsim 2 R_{\mathrm{e}}$, however, there appears to be a break that corresponds to the location of the $\mathrm{H} \alpha$ extension.

The estimated metallicity profile of this system, however, is highly uncertain, as indicated by the large error bars from IZI, which, unlike the $[\mathrm{N} \mathrm{II}] /[\mathrm{O} \mathrm{II}]$ calibration, considers multiple strong emission lines (see Section 2). These large error bars from IZI likely reflect the contrasting diagnostic line ratios, which may be due to the blending of physically different emission regions caused by insufficient spatial resolution.

Another source of uncertainty is the DIG contribution to the emission-line ratios, which could produce artificial metallicity gradients, especially in the outskirts of the system (K. Zhang et al. 2016, in preparation). While we argue that DIG is not the major component of this system in Section 4.1, we cannot rule out minor DIG contributions that could affect the estimated metallicity gradients of this system.

The ionized gas kinematics of this system is presented in Figure 3, with $v_{\text {peak }}$ displayed in panel (a) and $W_{80}$ in panel (b); again, we superimpose the $\mathrm{H} \alpha$ flux contours and highlight the two spaxels from Figure 1(b). Figure 3(a) reveals an asymmetric gradient that ranges from $v_{\text {peak }} \approx-140 \mathrm{~km} \mathrm{~s}^{-1}$ at the $\mathrm{H} \alpha$ extension to $v_{\text {peak }} \approx 40 \mathrm{~km} \mathrm{~s}^{-1}$ at the right side of the host galaxy. Figure 3(b) shows typical values of $W_{80} \approx 230 \mathrm{~km} \mathrm{~s}^{-1}$, with areas of enhanced $W_{80}$ in the $\mathrm{H} \alpha$ extension and the outer envelope of the $\mathrm{H} \alpha$ flux distribution. The velocity profile of the highlighted spaxels in Figure 3(a) is shown in Figure 3(c), with $R_{\mathrm{e}}$ of the host galaxy marked by the vertical dashed lines. $\left|v_{\text {peak }}\right|$ rises smoothly out to $\pm 5^{\prime \prime}$, where it starts to flatten. Beyond $-5^{\prime \prime}$, however, there is an irregular wiggle in $v_{\text {peak }}$ that corresponds to boosted $W_{80}$ in the $\mathrm{H} \alpha$ extension.

The [N II] and [S II] BPT (Baldwin et al. 1981) diagrams for this system are presented in Figures 4(a) and (b), respectively. We only consider spaxels with emission lines that have 
Table 1

Galaxy Properties

\begin{tabular}{|c|c|c|c|c|c|c|c|c|c|c|c|}
\hline MaNGA-ID & Plate-IFU & $\begin{array}{c}\text { R.A. } \\
\text { (J2000.0 deg) }\end{array}$ & $\begin{array}{c}\text { Decl. } \\
\text { (J2000.0 deg) }\end{array}$ & $z^{\mathrm{a}}$ & $\begin{array}{c}\log M_{*}{ }^{\mathrm{b}} \\
\left(M_{\odot}\right)\end{array}$ & $u-r^{c}$ & $\begin{array}{l}\log \mathrm{SFR}^{\mathrm{d}} \\
\left(M_{\odot} \mathrm{yr}^{-1}\right)\end{array}$ & $\begin{array}{c}\log \Sigma_{\mathrm{SFR}}{ }^{\mathrm{e}} \\
\left(M_{\odot} \mathrm{yr}^{-1} \mathrm{kpc}^{-2}\right)\end{array}$ & $\begin{array}{c}R_{\mathrm{e}}^{\mathrm{f}} \\
(\operatorname{arcsec})\end{array}$ & $\begin{array}{c}R_{\mathrm{e}} \\
(\mathrm{kpc})\end{array}$ & $n^{\mathrm{g}}$ \\
\hline $1-113700$ & $8618-12703$ & 319.45182 & 11.66059 & 0.038 & 9.77 & 2.05 & 0.22 & -0.37 & 2.6 & 2.0 & 3.7 \\
\hline
\end{tabular}

Notes.

${ }^{\text {a }}$ Spectroscopic redshift from NSA catalog.

${ }^{\mathrm{b}}$ Galaxy stellar mass from MPA-JHU DR7 data release.

${ }^{c}$ Rest-frame $u-r$ color from NSA catalog.

${ }^{\mathrm{d}}$ Fiber star formation rate from MPA-JHU DR7 data release.

${ }^{\mathrm{e}}$ Fiber star formation rate surface density using the MPA-JHU DR7 data release.

${ }^{\mathrm{f}}$ Effective radius from GALFIT (Peng et al. 2002).

${ }^{\mathrm{g}}$ Galaxy Sérsic index from GALFIT (Peng et al. 2002).

$S / N>3$ and overlay curves from Kewley et al. (2006) that are used to separate different classes of galaxies. Figures 4(c) and (d) display the resolved [N II] and [S II] BPT diagrams, respectively, i.e., these maps color-code each spaxel according to its location in their respective BPT diagrams. We overplot the $\mathrm{H} \alpha$ flux contours and highlight the same two spaxels in Figure 1(b). Figure 4 demonstrates that almost all spaxels have HII line ratios.

\section{DISCUSSION}

In this section, we discuss the nature of the $\mathrm{H} \alpha$ extension.

\subsection{Diffuse Ionized Gas}

The presence of warm, ionized gas in the outskirts of galaxies has been commonly referred to as DIG (Hoyle \& Ellis 1963; Reynolds 1985). The most pronounced characteristics of the DIG are the elevated [N II] $/ \mathrm{H} \alpha$ and [S II] $/ \mathrm{H} \alpha$ line ratios compared to H II regions (e.g., Monnet 1971; Rand et al. 1990; Rand 1997, 1998; Haffner et al. 1999; Tüllmann et al. 2000; Collins \& Rand 2001; Otte et al. 2001, 2002; Hoopes \& Walterbos 2003; Wood \& Mathis 2004; Voges \& Walterbos 2006; Rand et al. 2008; Haffner et al. 2009; Reynolds et al. 2012), which may be due to secondary ionization/heating sources (e.g., Reynolds \& Cox 1992; Reynolds et al. 1999). Thus, comparing the line ratios of the $\mathrm{H} \alpha$ extension to that of $\mathrm{H}$ II regions would constrain whether the $\mathrm{H} \alpha$ extension is DIG.

Ideally, we would identify individual H II regions in our system in order to conduct this comparison. However, the spatial resolution of the MaNGA data is too coarse-the FWHM is 2 "! 5 , which corresponds to $\approx 1.9 \mathrm{kpc}$ at $z=0.038$ (the host galaxy's redshift), which is more than an order of magnitude larger than typical H II regions (1-100 pc; Kennicutt 1984; Hunt \& Hirashita 2009). Therefore, every spaxel in our data contains contributions from both $\mathrm{H}$ II regions and DIG.

One way to constrain the DIG contribution of the $\mathrm{H} \alpha$ extension is by comparing its line ratios to that of the center of the host galaxy, where the $\mathrm{H} \alpha$ surface brightness is the highest and the DIG contribution is the lowest (Ferguson et al. 1996). However, since line ratios are also dependent on metallicity and ionization parameter (Dopita et al. 2000, 2013; Kewley \& Dopita 2002; Kewley et al. 2006) —which are likely to be different at the center compared to the $\mathrm{H} \alpha$ extension-another way to constrain the DIG contribution is to compare the line ratios of the $\mathrm{H} \alpha$ extension to the line ratios of the surrounding regions.
We present this line ratio comparison in Figure 5, all of the panels of which have been corrected for reddening according to Section 2. Figures 5(a)-(c) show the log line ratio maps of $[\mathrm{N} \mathrm{II}] / \mathrm{H} \alpha,[\mathrm{O} \mathrm{III]} / \mathrm{H} \alpha$, and $[\mathrm{S} \mathrm{II}] / \mathrm{H} \alpha$, and Figures 5(e)-(f) show the $\log$ line ratio maps of $[\mathrm{O}$ II] $] / \mathrm{H} \alpha$ and $[\mathrm{O} \mathrm{III]} / \mathrm{H} \beta$; the white contours represent the $\mathrm{H} \alpha$ flux distribution. Figure 5(d) displays the $[\mathrm{N} \mathrm{II}] / \mathrm{H} \alpha$ (filled circles), $[\mathrm{O} \mathrm{III}] / \mathrm{H} \alpha$ (open triangles), and $[\mathrm{S} \mathrm{II}] / \mathrm{H} \alpha$ (asterisks) line ratio profiles as traced by the highlighted spaxels in Figures 5(a)-(c), while Figure $5(\mathrm{~g})$ presents the $[\mathrm{O} \mathrm{II}] / \mathrm{H} \alpha$ (filled stars), $[\mathrm{O} \mathrm{III]} / \mathrm{H} \beta$ (open squares), and [S II]/[N II] (open diamonds) line ratio profiles as traced by the highlighted spaxels in Figures 5(e)-(f); the black solid line represents the $\mathrm{H} \alpha$ surface brightness profile over the same highlighted spaxels.

Figure 5 indicates a complicated situation. While Figures 5(d) and (g) show that $[\mathrm{N}$ II $] / \mathrm{H} \alpha$ generally decreases with more negative offsets from the center of the galaxy, i.e., toward the direction of the $\mathrm{H} \alpha$ extension, the $[\mathrm{S} \mathrm{II}] / \mathrm{H} \alpha$, [O III] $/$ $\mathrm{H} \alpha$, $[\mathrm{O} \mathrm{II}] / \mathrm{H} \alpha$, and $[\mathrm{O} \mathrm{III}] / \mathrm{H} \beta$ generally increase along the same direction. These enhancing line ratios toward the $\mathrm{H} \alpha$ extension are consistent with DIG, but the decreasing [N II]/ $\mathrm{H} \alpha$ line ratio toward the $\mathrm{H} \alpha$ extension is not consistent with DIG (e.g., Hoopes \& Walterbos 2003; Voges \& Walterbos 2006; Zhang et al. 2016, submitted). The latter trend is particularly striking since $[\mathrm{N} \mathrm{II}] / \mathrm{H} \alpha$ is sensitive to the temperature of the gas and has been used to infer that the DIG is about $\sim 2000 \mathrm{~K}$ hotter than $\mathrm{H}$ II regions-a defining characteristic of DIG (Haffner et al. 1999; Madsen et al. 2006; Haffner et al. 2009; Reynolds et al. 2012).

Figure 5(d) also shows that the $[\mathrm{N} \mathrm{II]} / \mathrm{H} \alpha$ profile displays a dip around an offset of $-5^{\prime \prime}$, which corresponds to the location of the $\mathrm{H} \alpha$ extension, while the $[\mathrm{S}$ II] $/ \mathrm{H} \alpha$ profile and the $\mathrm{H} \alpha$ surface brightness profile display a flattening at the same spot. Since the regions surrounding the $\mathrm{H} \alpha$ extension should have a similar metallicity and ionization parameter, the fact that the $\mathrm{H} \alpha$ extension does not display enhanced [N II] $/ \mathrm{H} \alpha$ and [S II]/ $\mathrm{H} \alpha$ compared to its surrounding regions indicates that the $\mathrm{H} \alpha$ extension is not dominated by DIG. However, since the $\mathrm{S} / \mathrm{N}$ is low at the outskirts, the comparison of the line ratios at the center of the $\mathrm{H} \alpha$ extension (at $\sim-5^{\prime \prime}$ ) to those at the outermost regions is more uncertain.

Another defining characteristic of DIG is its low density ( $\sim 10^{-3} \mathrm{~cm}^{-3}$; e.g., Haffner et al. 2009). Hence, another way to constrain the DIG contribution is to probe the gas density of the system with the [S II] $\lambda 6717 /[\mathrm{S}$ II] $\lambda 6731$ line ratio (Osterbrock \& Ferland 2006). We present the [S II] $\lambda 6717 /$ [S II] $\lambda 6731$ line ratio map in Figure 6(a) (with the $\mathrm{H} \alpha$ contours superimposed and the same spaxels from Figure 1(b) 

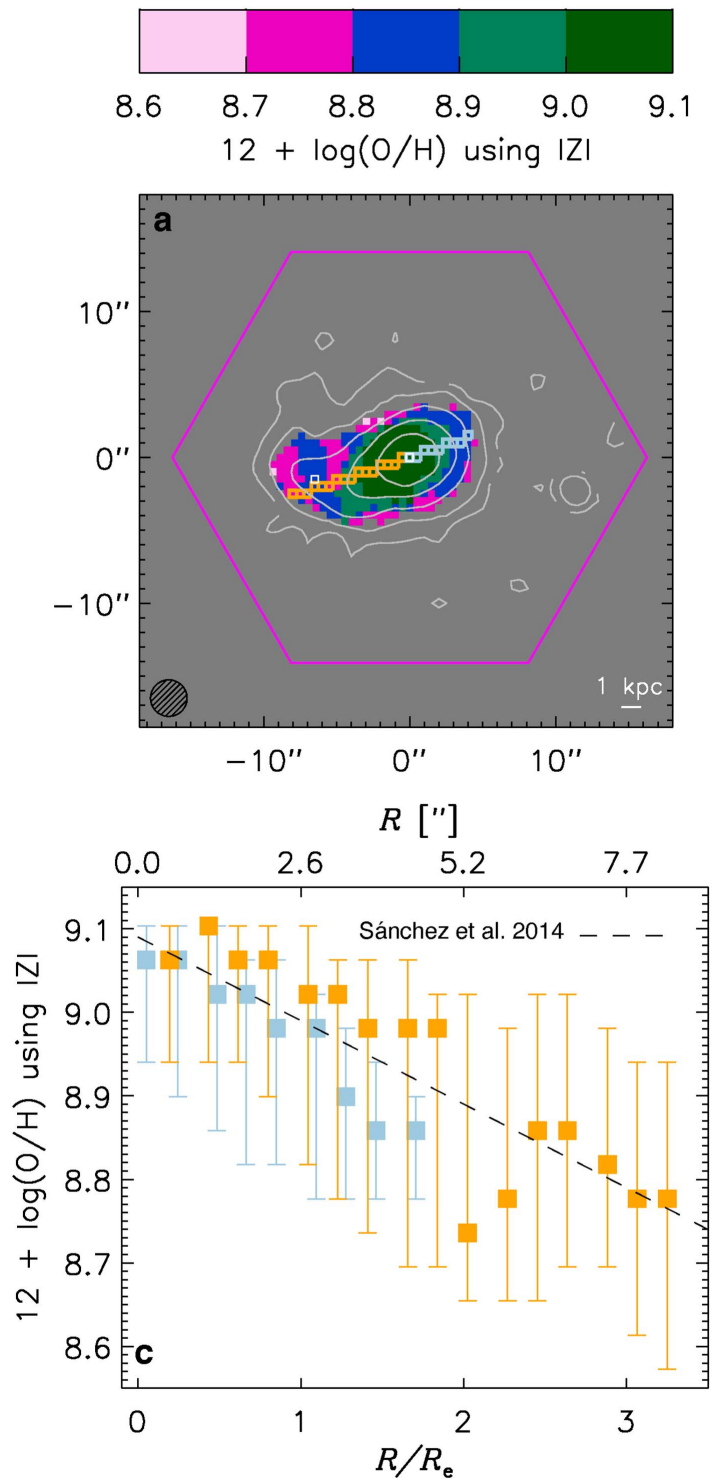
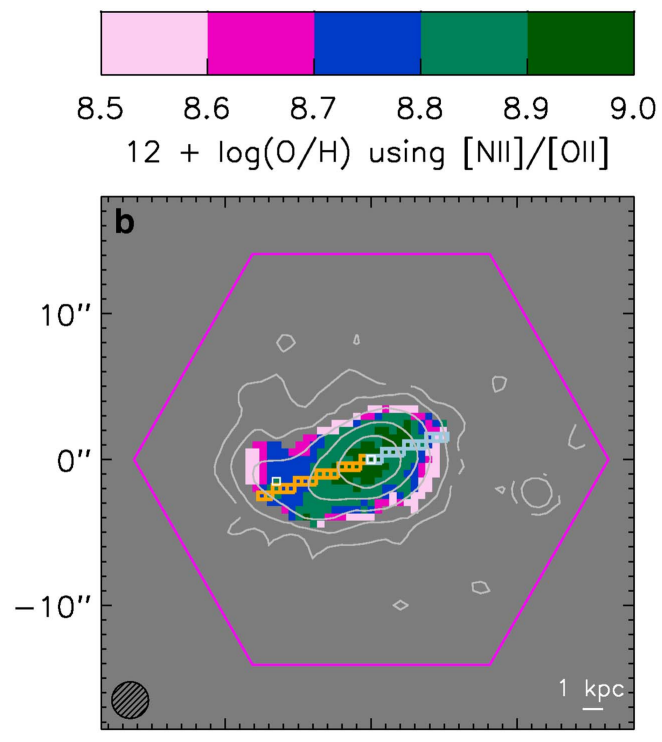

$-10^{\prime \prime} \quad 0^{\prime \prime} \quad 10^{\prime \prime}$

$$
R["]
$$

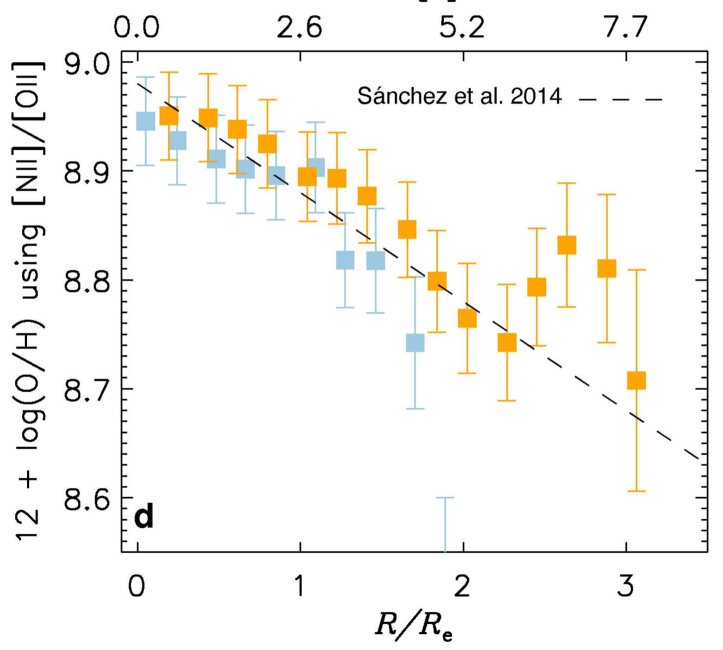

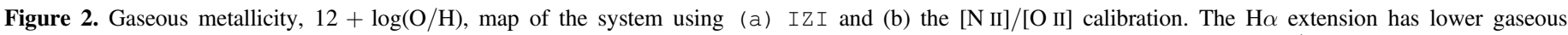

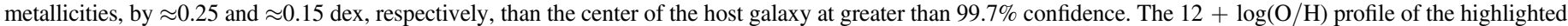

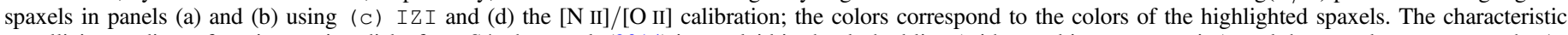

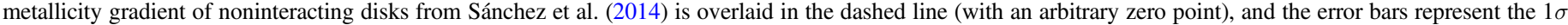
uncertainties.

highlighted in white) and the histogram of the [S II] $\lambda 6717 /$ [S II] $\lambda 6731$ line ratios for various regions in Figure 6(b).

The [S II] $\lambda 6717 /[$ S II] $\lambda 6731$ line ratios of the $\mathrm{H} \alpha$ circle range from 1.0 to 1.6 , which corresponds to electron densities, $n_{\mathrm{e}}$, of 500 to $<10 \mathrm{~cm}^{-3}$, respectively (Proxauf et al. 2014). Even though the leftmost edge of the $\mathrm{H} \alpha$ circle shows electron densities that are less than $10 \mathrm{~cm}^{-3}$, that a large area of the $\mathrm{H} \alpha$ extension contains dense gas with $n_{\mathrm{e}} \sim 500 \mathrm{~cm}^{-3}$ strongly indicates that the $\mathrm{H} \alpha$ extension is not dominated by DIG.

\subsection{Gas Accretion}

Alternatively, the $\mathrm{H} \alpha$ extension could be a sign of gas accretion (Sancisi et al. 2008; Rubin et al. 2012; Bouché et al. 2013, 2016; Sánchez Almeida et al. 2014, 2015), which can manifest through a variety of ways, e.g., cold flows (the properties of this galaxy, $\log M_{*} / \mathrm{M}_{\odot}=9.77$ and $z=0.038$, suggest cold flows rather than hot flows; Kereš et al. 2005, 2009b; Dekel \& Birnboim 2006; Dekel et al. 2009; Danovich et al. 2015; Stewart et al. 2016), recycled wind material (Shapiro \& Field 1976; Bregman 1980; Fraternali \& Binney 2008; Oppenheimer \& Davé 2008; Kereš et al. 2009a; Oppenheimer et al. 2010; Hopkins et al. 2014), or a gas-rich low-surface-brightness (LSB) dwarf galaxy (e.g., Fischer et al. 2015; Leaman et al. 2015).

The supersolar gaseous metallicities of this $\mathrm{H} \alpha$ extension (see Figure 2), however, are inconsistent with gas accretion through cold flows from the intergalactic medium (IGM) since this type of accreting gas is expected to be very metal-poor (Davé et al. 2011; Joung et al. 2012; van de Voort \& Schaye 2012).

The $\mathrm{H}$ II line ratios of the $\mathrm{H} \alpha$ extension indicate that star formation is the primary source of ionization (see Figure 4), suggesting that the $\mathrm{H} \alpha$ extension is an accreting gas-rich LSB dwarf galaxy. Assuming that this $\mathrm{H} \alpha$ extension is indeed a LSB 

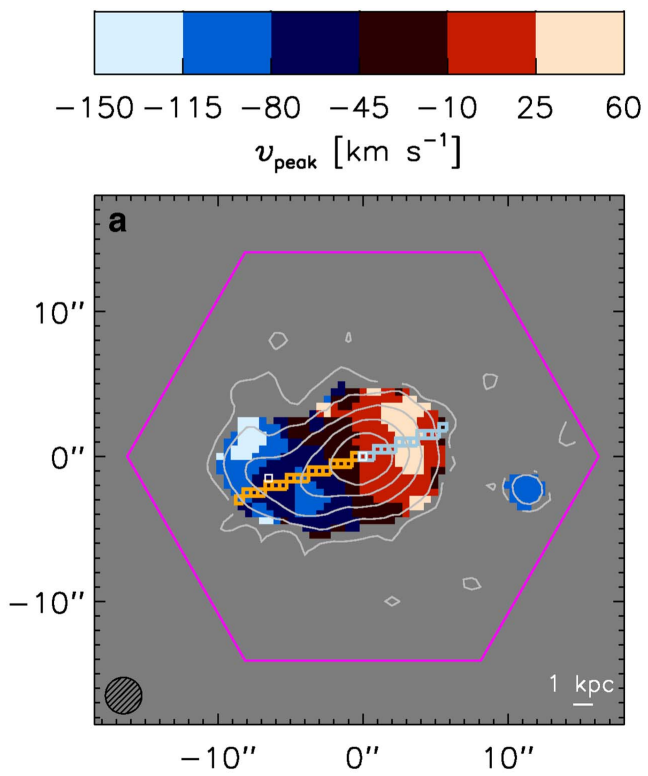
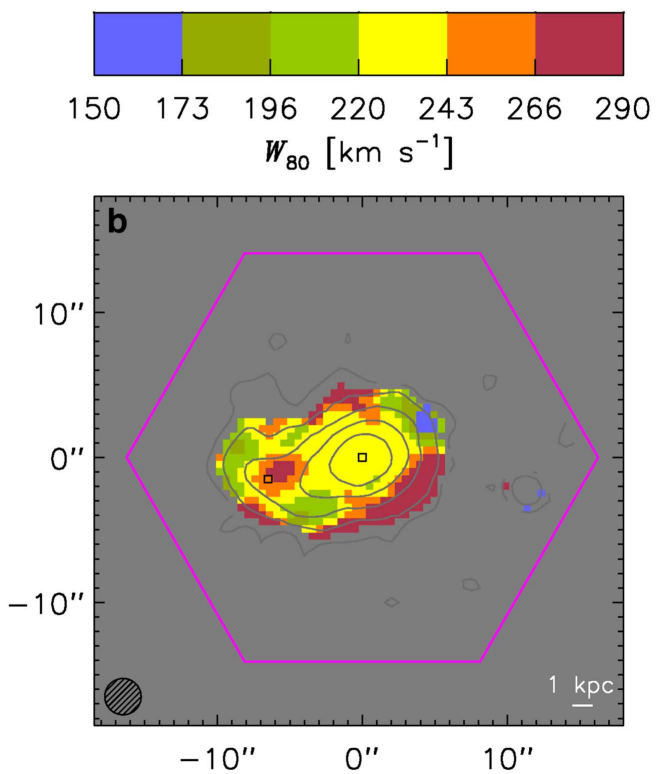

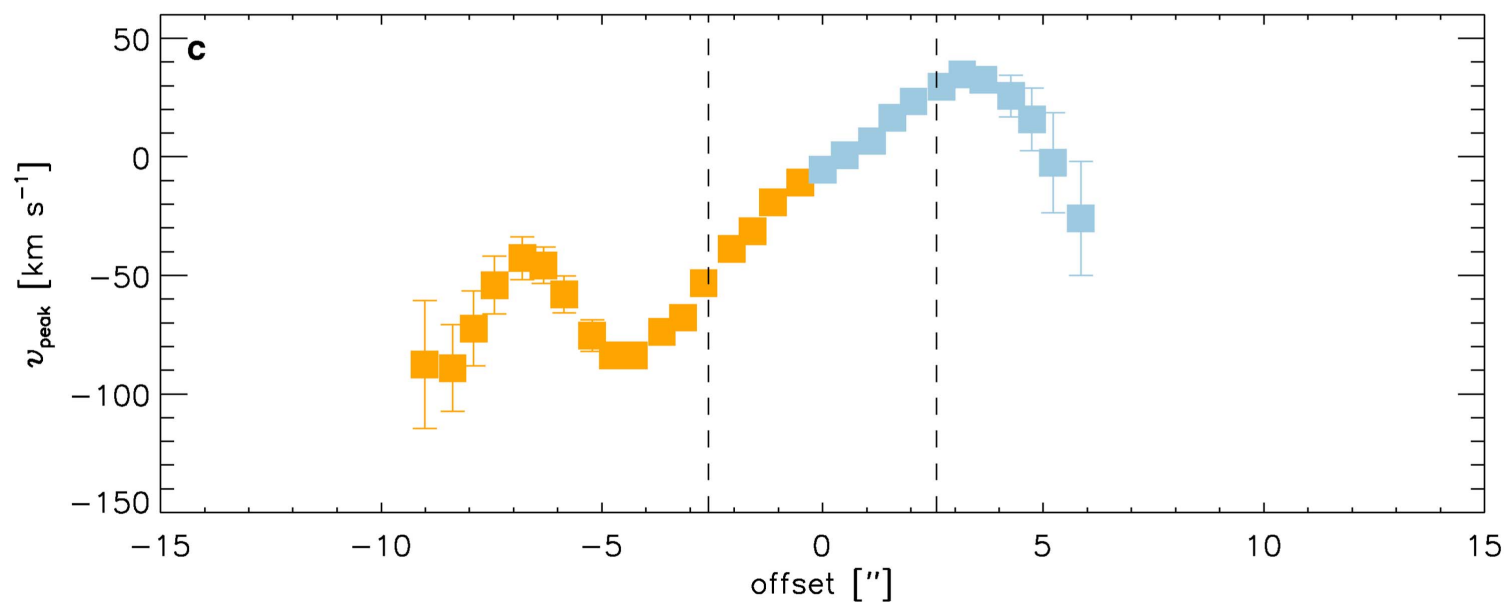

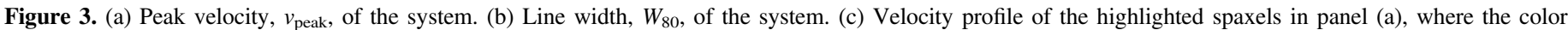

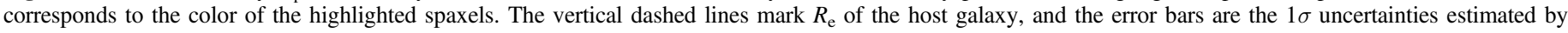

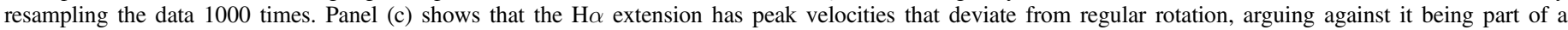
skewed disk.

dwarf galaxy, we estimate a stellar mass upper limit of $M_{*} \sim 10^{7} M_{\odot}$.

To obtain this limit, we first sum the $g$-band flux of every pixel in the $\mathrm{H} \alpha$ circle using SDSS DR7; we multiply this sum by 3 and take it as the upper limit of the $g$-band flux. Selecting SDSS DR7 galaxies at $0.02<z<0.04$, we plot their mass-tolight ratios $(M / L)$ as a function of rest-frame $g-r$ color using the MPA-JHU stellar masses and galaxy $g$ - and $r$-band absolute magnitudes from GIM2D (Simard et al. 2011) and then use the typical $M / L$ of star-forming galaxies $(0.2<g-r<0.4)$ to estimate the stellar mass upper limit of the $\mathrm{H} \alpha$ extension, which yields $M_{*} \sim 10^{7} M_{\odot}$.

A galaxy with such a low stellar mass, however, is unlikely to have the supersolar gaseous metallicities that are observed in the $\mathrm{H} \alpha$ extension (Berg et al. 2012; Jimmy et al. 2015), indicating that the $\mathrm{H} \alpha$ complex is probably not an accreting gas-rich LSB dwarf galaxy.

Finally, the enhanced values of $W_{80}$ within the $\mathrm{H} \alpha$ extension (see Figure 3(b)) that coincide with an unusual $v_{\text {peak }}$ bump in the velocity profile (at $\sim-7^{\prime \prime}$ of Figure 3(c)) may suggest the presence of turbulence that could be caused by the interaction between accreting gas and the host galaxy. However, such large values of $W_{80}$ may also be an artifact caused by the coarse resolution of the MaNGA data, which could blend multiple components separated by small velocities and/or small distances. Future higher-resolution data will be needed to discern these possibilities.

Therefore, the gas accretion scenario that best matches the properties of the $\mathrm{H} \alpha$ extension is recycled wind material (Oppenheimer \& Davé 2008; Kereš et al. 2009a; Oppenheimer et al. 2010; Hopkins et al. 2014), which is predicted to be a critical process in shaping the galaxy stellar mass function at low stellar masses $\left(<5 \times 10^{10} M_{*} / M_{\odot} ; \quad\right.$ Oppenheimer et al. 2010; Hopkins et al. 2014).

\subsubsection{Gas Accretion Deficit}

If this $\mathrm{H} \alpha$ extension is indeed accreting gas, then an interesting application would be toward the gas accretion deficit. To elaborate, Sancisi et al. (2008) combined many nearby $\mathrm{H}$ I studies to show that the visible $\mathrm{H}$ I gas accretion rate 

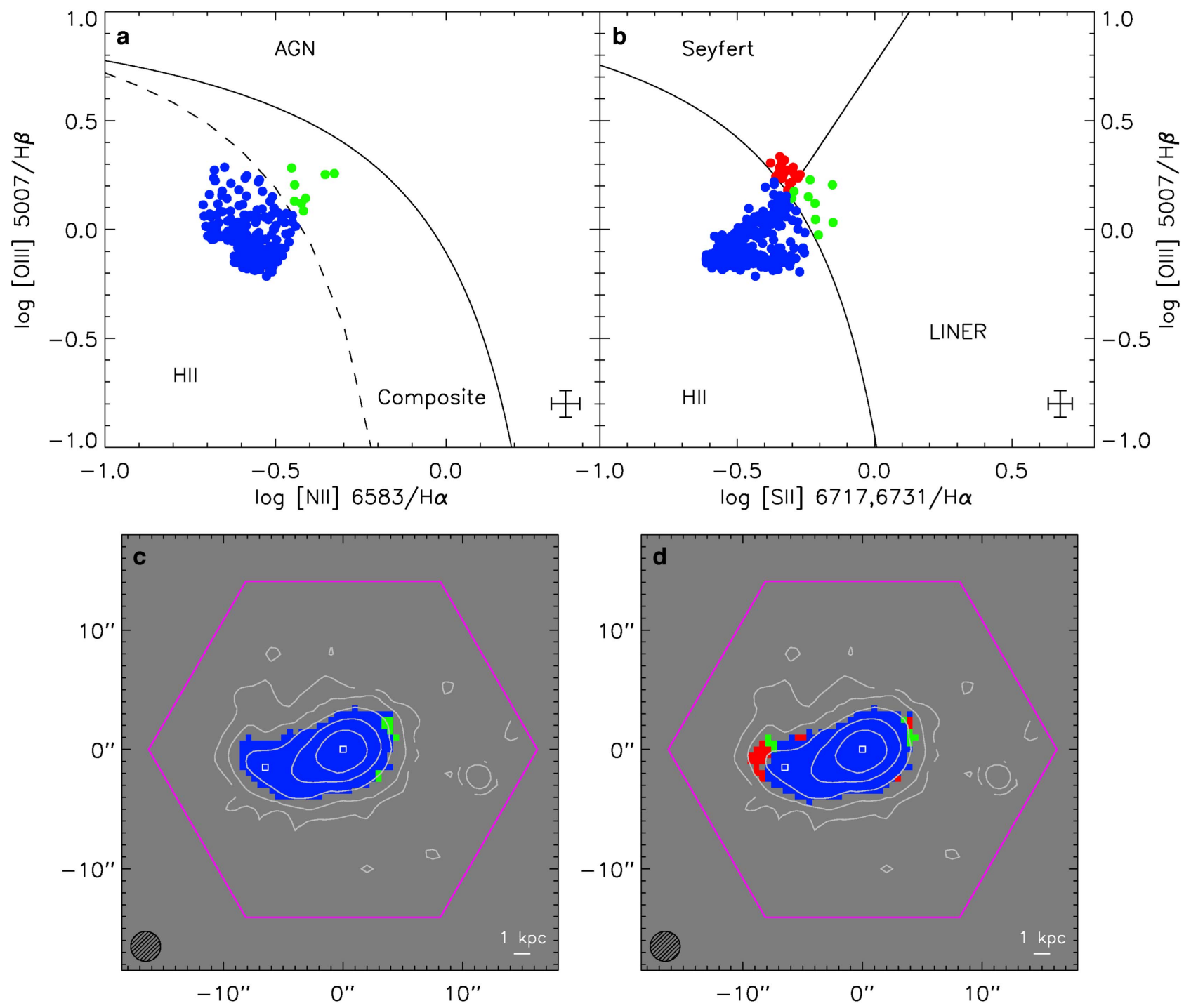

Figure 4. (a, b) [N II] and [S II] BPT diagrams, respectively; the lower right error bars represent the typical $1 \sigma$ measurement errors. (c, d) Resolved [N II] and [S II] BPT diagrams, respectively, i.e., every spaxel is color-coded according to its location in the BPT diagrams above. Almost all the spaxels of this system have line ratios in the $\mathrm{H}$ II regime.

in the local universe is only $\sim 0.2 M_{\odot} \mathrm{yr}^{-1}$, which is about an order of magnitude too small to account for the current SFR of local star-forming galaxies $\left(\sim 1 M_{\odot} \mathrm{yr}^{-1}\right)$. These H I studies, however, only probe cold gas $(T \lesssim 100 \mathrm{~K})$. Simulations predict that most of the gas in cold flows has $T \gtrsim 10^{4} \mathrm{~K}$ upon entering the host galaxy (Joung et al. 2012; van de Voort \& Schaye 2012; Nelson et al. 2013). Thus, probing warm $\left(\sim 10^{4} \mathrm{~K}\right)$, ionized gas from the CGM may alleviate the gas accretion deficit.

To test this idea, we compare the potential warm gas accretion rate onto this galaxy with its SFR. We first calculate the ionized gas mass of the $\mathrm{H} \alpha$ circle using the following equation from Osterbrock \& Ferland (2006):

$$
\frac{M_{\text {ionized gas }}}{2.82 \times 10^{9} M_{\odot}}=\left(\frac{L_{\mathrm{H} \beta}}{10^{43} \mathrm{erg} \mathrm{s}^{-1}}\right)\left(\frac{n_{\mathrm{e}}}{100 \mathrm{~cm}^{-3}}\right)^{-1},
$$

where $L_{\mathrm{H} \beta}$ is the extinction-corrected $\mathrm{H} \beta$ luminosity and $n_{\mathrm{e}}$ is the electron density. We calculate $L_{\mathrm{H} \beta}$ by summing the extinction-corrected $\mathrm{H} \beta$ luminosities of every spaxel in the $\mathrm{H} \alpha$ circle, yielding $L_{\mathrm{H} \beta}=2.89 \times 10^{39} \mathrm{erg} \mathrm{s}^{-1}$. To estimate $n_{\mathrm{e}}$, we measure the median [S II] $\lambda 6717 /[\mathrm{S}$ II] $\lambda 6731$ ratio $(\approx 1.3$; see Figure $6(\mathrm{~b}))$ of the $\mathrm{H} \alpha$ circle to obtain $n_{\mathrm{e}} \approx 50 \mathrm{~cm}^{-3} ; \quad$ this calculation yields $M_{\text {ionized gas }} \approx 1.63 \times 10^{6} M_{\odot}$.

The time required for this $\mathrm{H} \alpha$ circle to accrete onto the host galaxy, $t_{\text {accretion }}$, is estimated by dividing the distance between the $\mathrm{H} \alpha$ circle and the host galaxy with the observed velocity of the $\mathrm{H} \alpha$ circle. This distance, i.e., the distance between the two highlighted spaxels in Figure 1(b), is $\approx 6$ " 67, which corresponds to $1.5 \times 10^{17} \mathrm{~km}$. The median $\left|v_{\text {peak }}\right|$ of the $\mathrm{H} \alpha$ circle is $\approx 80 \mathrm{~km} \mathrm{~s}^{-1}$. Hence, $t_{\text {accretion }} \approx 6 \times 10^{7} \mathrm{yr}$.

Therefore, the average warm gas accretion rate onto the host galaxy is $M_{\text {ionized gas }} / t_{\text {accretion }} \approx 0.03 M_{\odot} \mathrm{yr}^{-1}$. If this accreting warm gas were driving the current star formation in the host galaxy, we would expect for this warm gas accretion rate to be similar to the current SFR. However, the estimated star formation rate of the host galaxy is SFR $=1.67 M_{\odot} \mathrm{yr}^{-1}$, which is almost two orders of magnitude larger than the estimated warm gas accretion rate. Thus, warm gas accretion does not appear to solve the gas accretion deficit for this galaxy. 

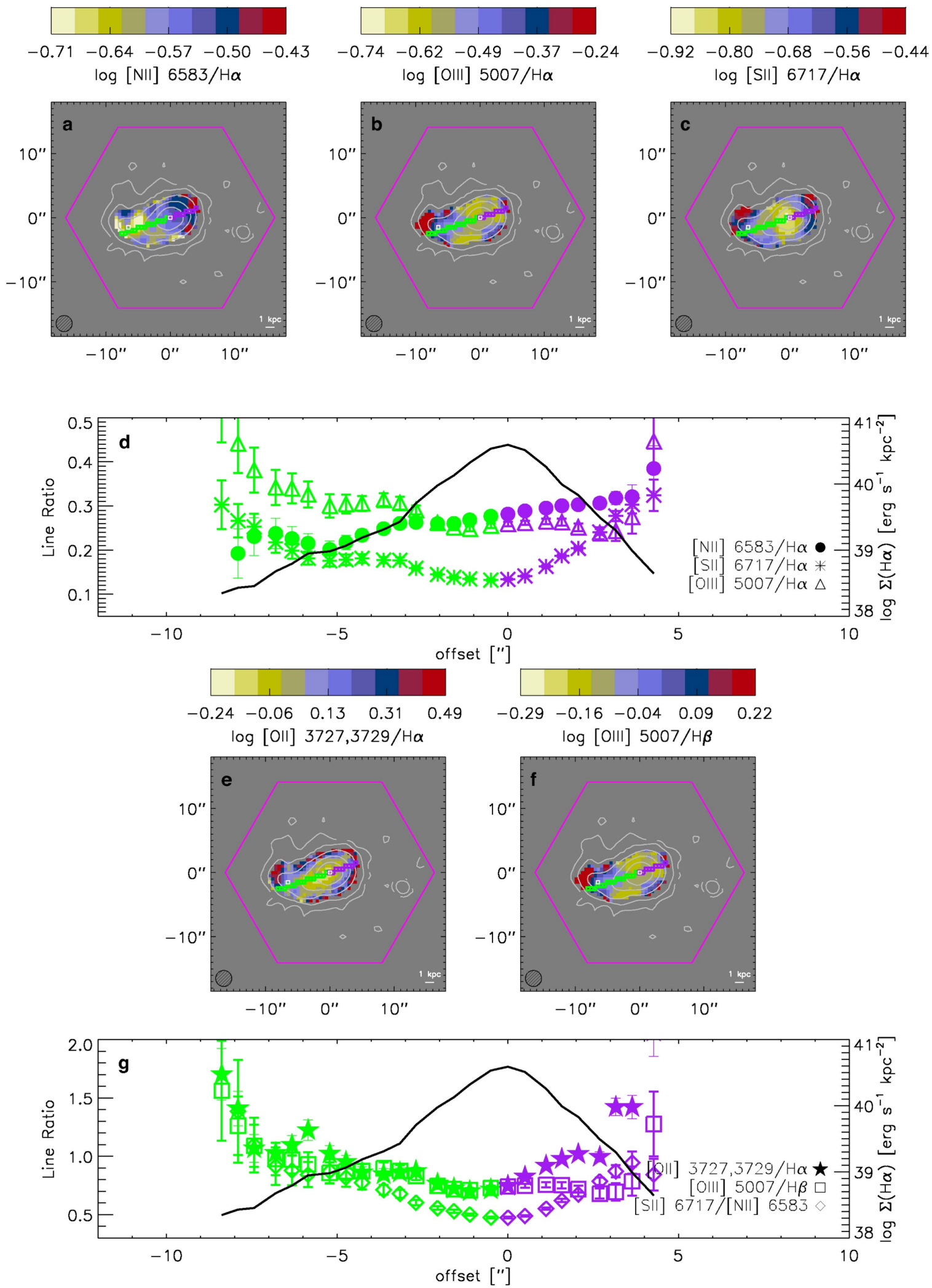

Figure 5. (a)-(c) The $\log [\mathrm{N}$ II $] / \mathrm{H} \alpha, \log [\mathrm{O}$ III $] / \mathrm{H} \alpha$, and $\log [\mathrm{S} \mathrm{II}] / \mathrm{H} \alpha$ line ratio maps. (d) Line ratio profiles of $[\mathrm{N} \mathrm{II]} / \mathrm{H} \alpha$, [O III] $/ \mathrm{H} \alpha$, and $[\mathrm{S} \mathrm{II]} / \mathrm{H} \alpha$ of the highlighted spaxels in panels (a)-(c); the colors correspond to the colors of the highlighted spaxels, and the error bars represent the $1 \sigma$ measurement error. The black line represents the $\mathrm{H} \alpha$ surface brightness profile across the same highlighted spaxels, with its values indicated by the $y$-axis on the right side. (e)-(f) The log [O II]/ $\mathrm{H} \alpha$ and $\log [\mathrm{O} \mathrm{III]} / \mathrm{H} \beta$ line ratio maps. (g) The line ratio profiles of $[\mathrm{O} \mathrm{III} / \mathrm{H} \alpha$, [O III] $/ \mathrm{H} \beta$, and $[\mathrm{S} \mathrm{II}] /[\mathrm{N} \mathrm{II}]$ of the highlighted spaxels in panels (e)-(f). We only consider spaxels with $\mathrm{S} / \mathrm{N}>3$ for these emission lines and have corrected all these line ratios for reddening. 


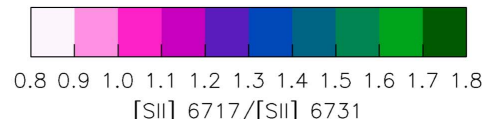
[SII] $6717 /[$ SII] 6731
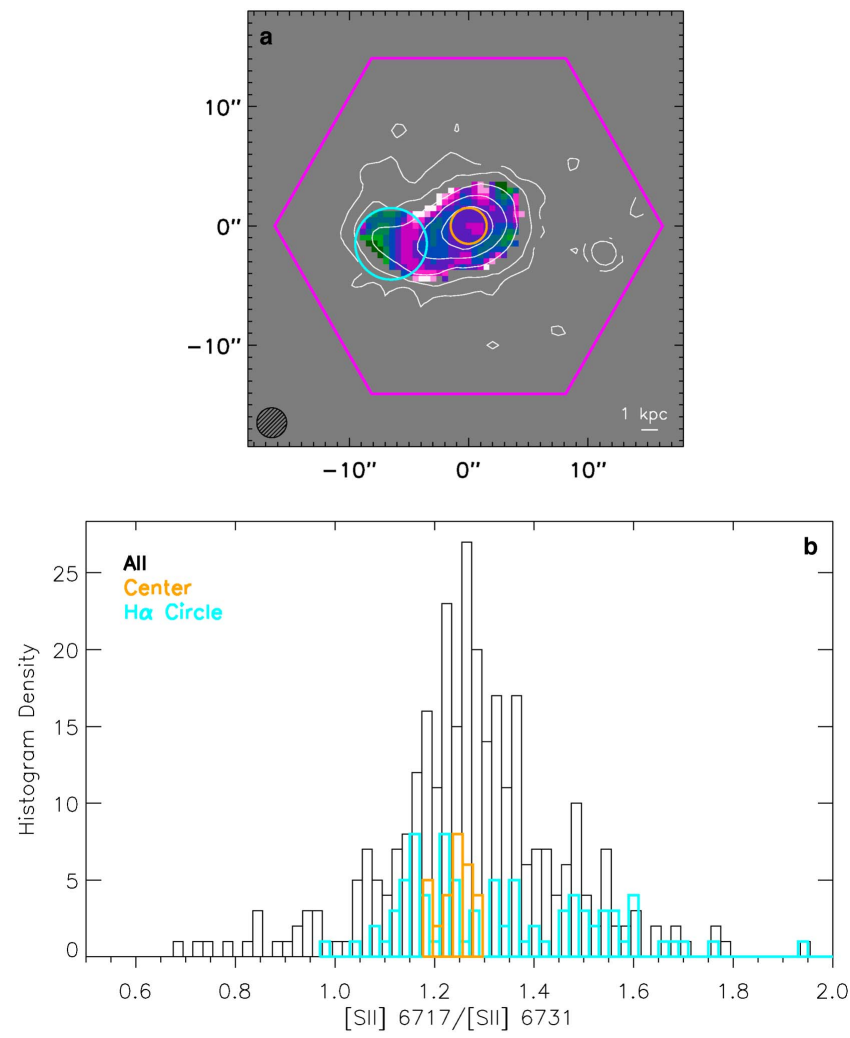

Figure 6. (a) [S II] 6717/[S II] 6731 line ratio map. (b) Histogram of the [S II] $\lambda 6717 /[\mathrm{S} \mathrm{II}] \lambda 6731$ line ratios of the entire system (black), the central region (orange, as indicated by the orange circle in the top panel), and the $\mathrm{H} \alpha$ circle (cyan).

However, since this galaxy is starbursting, which is defined to be a short period of intense star formation, perhaps it is unsurprising that the warm gas accretion does not sustain the current star formation rate. Moreover, this estimated warm gas accretion rate may be a lower limit since we do not account for transverse motions, meaning that the true velocity could be higher, nor do we account for the possibility of more DIG that is below our detection limit.

\subsection{Other Possibilities}

Another possibility for this $\mathrm{H} \alpha$ complex is that it is an outflow. If it were an outflow from a starburst, though, we would expect for its $12+\log (\mathrm{O} / \mathrm{H})$ values to be the same as, or higher than, the center of the host galaxy, where the metalenriching stellar feedback would have likely originated. Instead, we find that the $\mathrm{H} \alpha$ extension has lower $12+\log$ $(\mathrm{O} / \mathrm{H})$, arguing against an outflow. Another possibility is that it is gas disrupted and/or stripped by environmental processes, e.g., mergers or ram pressure stripping (Gunn \& Gott 1972). However, since the host galaxy shows no signs of interaction and does not appear to be in a large group, we find this explanation unlikely. Moreover, with a galaxy Sérsic index of $n=3.7$, unusual gaseous metallicity profile, and irregular rotation curve, it is also unlikely that this $\mathrm{H} \alpha$ extension is simply part of the host galaxy as a dim, lopsided disk.
Finally, the elongated, asymmetric $\mathrm{H} \alpha$ flux distribution of the system (see Figure 1) resembles the morphologies of tadpole galaxies (van den Bergh et al. 1996; Elmegreen et al. 2005, 2007, 2012; Sánchez Almeida et al. 2013; Straughn et al. 2015), suggesting that this $\mathrm{H} \alpha$ extension may be part of a tadpole galaxy. However, in addition to their cometary morphology, another common property of tadpole galaxies is their bright, blue colors that are indicative of recent bursts of intense star formation (Elmegreen \& Elmegreen 2010; Elmegreen et al. 2012). Since the $\mathrm{H} \alpha$ extension in our system has no optical or ultraviolet (UV) counterpart, it is unlikely that our system is a tadpole galaxy. Moreover, the supersolar gaseous metallicities of our system are inconsistent with the very metalpoor gaseous metallicities found in local tadpole galaxies (Sánchez Almeida et al. 2013).

\section{CONCLUSION}

In this paper, we present the serendipitous observation of an ionized gas structure that protrudes out of a dusty, starbursting galaxy. Our analysis indicates that this ionized gas complex is most consistent with gas accretion through recycled wind material.

To better understand the nature of this phenomenon, and to constrain the importance of gas accretion through recycled wind material, we need to find more of these extended gas complexes. However, the current MaNGA sample has not observed any other isolated galaxy with an adequately sized integral field unit ( $\gtrsim 6.3 R_{\mathrm{e}}$ ) to allow such a search. We hope to address this issue with future MaNGA releases.

We thank Kevin Bundy, Kyle Westfall, and Matthew Bershady for helpful comments and discussions. We also thank the anonymous referee for a constructive report that improved this work.

Funding for the Sloan Digital Sky Survey IV has been provided by the Alfred P. Sloan Foundation, the U.S. Department of Energy Office of Science, and the Participating Institutions. SDSS-IV acknowledges support and resources from the Center for High-Performance Computing at the University of Utah. The SDSS Web site is www.sdss.org. SDSS-IV is managed by the Astrophysical Research Consortium for the Participating Institutions of the SDSS Collaboration, including the Brazilian Participation Group, the Carnegie Institution for Science, Carnegie Mellon University, the Chilean Participation Group, the French Participation Group, Harvard-Smithsonian Center for Astrophysics, Instituto de Astrofísica de Canarias, Johns Hopkins University, Kavli Institute for the Physics and Mathematics of the Universe (IPMU)/University of Tokyo, Lawrence Berkeley National Laboratory, Leibniz Institut für Astrophysik Potsdam (AIP), Max-Planck-Institut für Astronomie (MPIA Heidelberg), MaxPlanck-Institut für Astrophysik (MPA Garching), Max-PlanckInstitut für Extraterrestrische Physik (MPE), National Astronomical Observatory of China, New Mexico State University, New York University, University of Notre Dame, Observatário Nacional/MCTI, The Ohio State University, Pennsylvania State University, Shanghai Astronomical Observatory, United Kingdom Participation Group, Universidad Nacional Autónoma de México, University of Arizona, University of Colorado Boulder, University of Oxford, University of Portsmouth, University of Utah, University of Virginia, University of Washington, University of Wisconsin, Vanderbilt University, 
and Yale University. D.B. is supported by grant RSCF-14-2200041. A.W. acknowledges support from a Leverhulme Early Career Fellowship. J.H.K. acknowledges financial support from the Spanish Ministry of Economy and Competitiveness (MINECO) under grant number AYA2013-41243-P and thanks the Astrophysics Research Institute of Liverpool John Moores University for their hospitality, and the Spanish Ministry of Education, Culture and Sports for financial support of his visit there, through grant number PR2015-00512.

\section{REFERENCES}

Abazajian, K. N., Adelman-McCarthy, J. K., Agüeros, M. A., et al. 2009, ApJS, 182, 543

Asplund, M., Grevesse, N., Sauval, A. J., \& Scott, P. 2009, ARA\&A, 47, 481 Baldwin, J. A., Phillips, M. M., \& Terlevich, R. 1981, PASP, 93, 5 Belfiore, F., Maiolino, R., Bundy, K., et al. 2015, MNRAS, 449, 867 Berg, D. A., Skillman, E. D., Marble, A. R., et al. 2012, ApJ, 754, 98 Bergeron, J. 1986, A\&A, 155, L8

Blanc, G. A., Kewley, L., Vogt, F. P. A., \& Dopita, M. A. 2015, ApJ, 798, 99 Bouché, N., Finley, H., Schroetter, I., et al. 2016, ApJ, 820, 121

Bouché, N., Murphy, M. T., Kacprzak, G. G., et al. 2013, Sci, 341, 50 Bregman, J. N. 1980, ApJ, 236, 577

Brinchmann, J., Charlot, S., White, S. D. M., et al. 2004, MNRAS, 351, 1151 Bruzual, G., \& Charlot, S. 2003, MNRAS, 344, 1000

Bundy, K., Bershady, M. A., Law, D. R., et al. 2015, ApJ, 798, 7

Cappellari, M., \& Emsellem, E. 2004, PASP, 116, 138

Charlot, S., \& Longhetti, M. 2001, MNRAS, 323, 887

Collins, J. A., \& Rand, R. J. 2001, ApJ, 551, 57

Danovich, M., Dekel, A., Hahn, O., Ceverino, D., \& Primack, J. 2015, MNRAS, 449, 2087

Davé, R., Finlator, K., \& Oppenheimer, B. D. 2011, MNRAS, 416, 1354

Dekel, A., \& Birnboim, Y. 2006, MNRAS, 368, 2

Dekel, A., Birnboim, Y., Engel, G., et al. 2009, Natur, 457, 451

Dopita, M. A., Kewley, L. J., Heisler, C. A., \& Sutherland, R. S. 2000, ApJ, 542,224

Dopita, M. A., Sutherland, R. S., Nicholls, D. C., Kewley, L. J., \& Vogt, F. P. A. 2013, ApJS, 208, 10

Drory, N., MacDonald, N., Bershady, M. A., et al. 2015, AJ, 149, 77

Elmegreen, B. G., \& Elmegreen, D. M. 2010, ApJ, 722, 1895

Elmegreen, D. M., Elmegreen, B. G., Ravindranath, S., \& Coe, D. A. 2007, ApJ, 658, 763

Elmegreen, D. M., Elmegreen, B. G., Rubin, D. S., \& Schaffer, M. A. 2005 , ApJ, 631, 85

Elmegreen, D. M., Elmegreen, B. G., Sánchez Almeida, J., et al. 2012, ApJ, 750,95

Ferguson, A. M. N., Wyse, R. F. G., Gallagher, J. S., III, \& Hunter, D. A. 1996, AJ, 111, 2265

Fischer, T. C., Crenshaw, D. M., Kraemer, S. B., et al. 2015, ApJ, 799, 234

Fitzpatrick, E. L. 1999, PASP, 111, 63

Fraternali, F., \& Binney, J. J. 2008, MNRAS, 386, 935

Gunn, J. E., \& Gott, J. R., III 1972, ApJ, 176, 1

Gunn, J. E., Siegmund, W. A., Mannery, E. J., et al. 2006, AJ, 131, 2332

Haffner, L. M., Dettmar, R.-J., Beckman, J. E., et al. 2009, RvMP, 81, 969

Haffner, L. M., Reynolds, R. J., \& Tufte, S. L. 1999, ApJ, 523, 223

Harrison, C. M., Alexander, D. M., Mullaney, J. R., \& Swinbank, A. M. 2014 MNRAS, 441, 3306

Hoopes, C. G., \& Walterbos, R. A. M. 2003, ApJ, 586, 902

Hopkins, P. F., Kereš, D., Oñorbe, J., et al. 2014, MNRAS, 445, 581

Hoyle, F., \& Ellis, G. R. A. 1963, AuJPh, 16, 1

Hunt, L. K., \& Hirashita, H. 2009, A\&A, 507, 1327

Jimmy, Tran, K.-V., Saintonge, A., et al. 2015, ApJ, 812, 98

Joung, M. R., Putman, M. E., Bryan, G. L., Fernández, X., \& Peek, J. E. G. 2012, ApJ, 759, 137

Kauffmann, G., Heckman, T. M., White, S. D. M., et al. 2003, MNRAS, 341,33

Kennicutt, R. C., Jr. 1984, ApJ, 287, 116

Kennicutt, R. C., Jr., Bresolin, F., \& Garnett, D. R. 2003, ApJ, 591, 801

Kennicutt, R. C., \& Evans, N. J. 2012, ARA\&A, 50, 531

Kereš, D., Katz, N., Davé, R., Fardal, M., \& Weinberg, D. H. 2009a, MNRAS, 396,2332
Kereš, D., Katz, N., Fardal, M., Davé, R., \& Weinberg, D. H. 2009b, MNRAS, 395, 160

Kereš, D., Katz, N., Weinberg, D. H., \& Davé, R. 2005, MNRAS, 363, 2

Kewley, L. J., \& Dopita, M. A. 2002, ApJS, 142, 35

Kewley, L. J., Groves, B., Kauffmann, G., \& Heckman, T. 2006, MNRAS, 372, 961

Lanzetta, K. M., Bowen, D. V., Tytler, D., \& Webb, J. K. 1995, ApJ, 442, 538

Law, D. R., Yan, R., Bershady, M. A., et al. 2015, AJ, 150, 19

Leaman, R., Erroz-Ferrer, S., Cisternas, M., \& Knapen, J. H. 2015, MNRAS, 450,2473

Levesque, E. M., Kewley, L. J., \& Larson, K. L. 2010, AJ, 139, 712

Li, C., Wang, E., Lin, L., et al. 2015, ApJ, 804, 125

Madsen, G. J., Reynolds, R. J., \& Haffner, L. M. 2006, ApJ, 652, 401

Miller, S. T., \& Veilleux, S. 2003, ApJ, 592, 79

Monnet, G. 1971, A\&A, 12, 379

Nelson, D., Vogelsberger, M., Genel, S., et al. 2013, MNRAS, 429, 3353

Oppenheimer, B. D., \& Davé, R. 2008, MNRAS, 387, 577

Oppenheimer, B. D., Davé, R., Kereš, D., et al. 2010, MNRAS, 406, 2325

Osterbrock, D. E., \& Ferland, G. J. 2006, in Astrophysics of Gaseous Nebulae and Active Galactic Nuclei, ed. D. E. Osterbrock \& G. J. Ferland. (2nd ed.; Sausalito, CA: Univ. Science Books)

Otte, B., Gallagher, J. S., III, \& Reynolds, R. J. 2002, ApJ, 572, 823

Otte, B., Reynolds, R. J., Gallagher, J. S., III, \& Ferguson, A. M. N. 2001, ApJ, 560, 207

Peng, C. Y., Ho, L. C., Impey, C. D., \& Rix, H.-W. 2002, AJ, 124, 266

Proxauf, B., Öttl, S., \& Kimeswenger, S. 2014, A\&A, 561, A10

Rand, R. J. 1997, ApJ, 474, 129

Rand, R. J. 1998, ApJ, 501, 137

Rand, R. J., Kulkarni, S. R., \& Hester, J. J. 1990, ApJL, 352, L1

Rand, R. J., Wood, K., \& Benjamin, R. A. 2008, ApJ, 680, 263

Reynolds, R. J. 1985, ApJ, 294, 256

Reynolds, R. J., \& Cox, D. P. 1992, ApJL, 400, L33

Reynolds, R. J., Haffner, L. M., Madsen, G. J., Wood, K., \& Hill, A. S. 2012, EAS Publications Series, 56, 213

Reynolds, R. J., Haffner, L. M., \& Tufte, S. L. 1999, ApJL, 525, L21

Rossa, J., \& Dettmar, R.-J. 2003, A\&A, 406, 493

Rubin, K. H. R., Prochaska, J. X., Koo, D. C., \& Phillips, A. C. 2012, ApJL, 747, L26

Salim, S., Rich, R. M., Charlot, S., et al. 2007, ApJS, 173, 267

Sánchez, S. F., Rosales-Ortega, F. F., Iglesias-Páramo, J., et al. 2014, A\&A, 563, A49

Sánchez Almeida, J., Elmegreen, B. G., Muñoz-Tuñón, C., et al. 2015, ApJL, 810, L15

Sánchez Almeida, J., Elmegreen, B. G., Muñoz-Tuñón, C., \& Elmegreen, D. M. 2014, A\&ARv, 22, 71

Sánchez Almeida, J., Muñoz-Tuñón, C., Elmegreen, D. M., Elmegreen, B. G., \& Méndez-Abreu, J. 2013, ApJ, 767, 74

Sancisi, R., Fraternali, F., Oosterloo, T., \& van der Hulst, T. 2008, A\&ARv, 15,189

SDSS Collaboration, Albareti, F. D., Allende Prieto, C., et al. 2016, arXiv: 1608.02013

Shapiro, P. R., \& Field, G. B. 1976, ApJ, 205, 762

Simard, L., Mendel, J. T., Patton, D. R., Ellison, S. L., \& McConnachie, A. W. 2011, ApJS, 196, 11

Smee, S. A., Gunn, J. E., Uomoto, A., et al. 2013, AJ, 146, 32

Stewart, K., Maller, A., Oñorbe, J., et al. 2016, arXiv:1606.08542

Straughn, A. N., Voyer, E. N., Eufrasio, R. T., et al. 2015, ApJ, 814, 97

Tremonti, C. A., Heckman, T. M., Kauffmann, G., et al. 2004, ApJ, 613, 898

Tüllmann, R., Dettmar, R.-J., Soida, M., Urbanik, M., \& Rossa, J. 2000, A\&A, 364, L36

Tumlinson, J., Thom, C., Werk, J. K., et al. 2011, Sci, 334, 948

van de Voort, F., \& Schaye, J. 2012, MNRAS, 423, 2991

van den Bergh, S., Abraham, R. G., Ellis, R. S., et al. 1996, AJ, 112, 359

Vazdekis, A., Ricciardelli, E., Cenarro, A. J., et al. 2012, MNRAS, 424, 157

Voges, E. S., \& Walterbos, R. A. M. 2006, ApJL, 644, L29

Werk, J. K., Prochaska, J. X., Thom, C., et al. 2013, ApJS, 204, 17

Werk, J. K., Prochaska, J. X., Tumlinson, J., et al. 2014, ApJ, 792, 8

Wilkinson, D. M., Maraston, C., Thomas, D., et al. 2015, MNRAS, 449,328

Wood, K., \& Mathis, J. S. 2004, MNRAS, 353, 1126

Yan, R., Newman, J. A., Faber, S. M., et al. 2006, ApJ, 648, 281

Yan, R., Tremonti, C., Bershady, M. A., et al. 2016, AJ, 151, 8

York, D. G., Adelman, J., Anderson, J. E., Jr., et al. 2000, AJ, 120, 1579 